\title{
Categories over quantum affine algebras and monoidal categorification
}

\author{
By Masaki KashiwarA, M.J.A., ${ }^{* 1), * 2), * 3)}$ Myungho KIM, ${ }^{* 4)}$ Se-jin $\mathrm{OH}^{* 5)}$ and Euiyong PARK ${ }^{* 6)}$ \\ (Contributed by Masaki KashiwarA, M.J.A., June 14, 2021)
}

\begin{abstract}
Let $U_{q}^{\prime}(\mathfrak{g})$ be a quantum affine algebra of untwisted affine $A D E$ type, and $\mathscr{C}_{\mathfrak{g}}^{0}$ the Hernandez-Leclerc category of finite-dimensional $U_{q}^{\prime}(\mathfrak{g})$-modules. For a suitable infinite sequence $\widehat{w}_{0}=\cdots s_{i_{-1}} s_{i_{0}} s_{i_{1}} \cdots$ of simple reflections, we introduce subcategories $\mathscr{C}_{\mathfrak{g}}^{[a, b]}$ of $\mathscr{C}_{\mathfrak{g}}^{0}$ for all $a \leqslant b \in \mathbf{Z} \sqcup\{ \pm \infty\}$. Associated with a certain chain $\mathfrak{C}$ of intervals in $[a, b]$, we construct a real simple commuting family $M(\mathfrak{C})$ in $\mathscr{C}_{\mathfrak{g}}^{[a, b]}$, which consists of Kirillov-Reshetikhin modules. The category $\mathscr{C}_{\mathfrak{g}}^{[a, b]}$ provides a monoidal categorification of the cluster algebra $K\left(\mathscr{C}_{\mathfrak{g}}^{[a, b]}\right)$, whose set of initial cluster variables is $[M(\mathfrak{C})]$. In particular, this result gives an affirmative answer to the monoidal categorification conjecture on $\mathscr{C}_{\mathfrak{g}}^{-}$by Hernandez-Leclerc since it is $\mathscr{C}_{\mathfrak{g}}^{[-\infty, 0]}$, and is also applicable to $\mathscr{C}_{\mathfrak{g}}^{0}$ since it is $\mathscr{C}_{\mathfrak{g}}^{[-\infty, \infty]}$.
\end{abstract}

Key words: Monoidal categorification; quantum affine algebra; cluster algebra; KirillovReshetikhin module; $T$-system.

1. Introduction. Let $U_{q}^{\prime}(\mathfrak{g})$ be a quantum affine algebra. The category $\mathscr{C}_{\mathfrak{g}}$ of finite-dimensional integrable modules over $U_{q}^{\prime}(\mathfrak{g})$ has been intensively studied due to its rich structure. For instances, every object $M$ in $\mathscr{C}_{\mathfrak{g}}$ has its left $M^{*}$ and right dual ${ }^{*} M$, and the $q$-characters of KirillovReshetikhin modules in $\mathscr{C}_{\mathfrak{g}}$ provide a solution of the $T$-system, a system of differential equations appearing in solvable lattice models $([3,5,18,19])$.

On the other hand, the cluster algebras were introduced by Fomin and Zelivinsky in [2] to investigate upper global bases and total positivity in an aspect of combinatorics.

Interestingly, it is proved in $[6,7,9]$ that the Grothendieck rings $K(\mathcal{C})$ of monoidal subcategories $\mathcal{C}=\mathscr{C}_{N} \quad\left(N \in \mathbf{Z}_{\geqslant 1}\right), \quad \mathscr{C}_{\mathcal{Q}}, \mathscr{C}_{\mathfrak{g}}^{-}$of $\mathscr{C}_{\mathfrak{g}}$ have cluster

2010 Mathematics Subject Classification. Primary 17B37, 81R50; Secondary 18D10.

*1) Kyoto University Institute for Advanced Study, Yoshida Ushinomiya-cho, Sakyo-ku, Kyoto 606-8501, Japan.

*2) Research Institute for Mathematical Sciences, Kyoto University, Kitashirakawa-Oiwakecho, Sakyo-ku, Kyoto 6068502, Japan.

*3) Korea Institute for Advanced Study, 85 Hoegiro, Dongdaemun-gu, Seoul 02455, Republic of Korea.

*4) Department of Mathematics, Kyung Hee University, 26 Kyungheedae-ro, Dongdaemun-gu, Seoul 02447, Republic of Korea.

*5) Department of Mathematics, Ewha Womans University, 52 Ewhayeodae-gil, Seodaemun-gu, Seoul 03760, Republic of Korea.

*6) Department of Mathematics, University of Seoul, 163 Seoulsiripdae-ro, Dongdaemun-gu, Seoul 02504, Republic of Korea. algebra structures $\mathscr{A}$, and conjectured that every cluster monomial corresponds to the isomorphism class of a real simple module in $\mathcal{C}$; that is, $\mathcal{C}$ is expected to be a monoidal categorification of $\mathscr{A}$. The conjectures for $\mathscr{C}_{N}\left(N \in \mathbf{Z}_{\geqslant 1}\right)$ of untwisted affine $A D E$ types are proved in $[6,8,20]$ and [23]. Also, the conjecture for the subcategory $\mathscr{C}_{\mathcal{Q}} \subset \mathscr{C}_{\mathfrak{g}}$, determined by a Q-data $\mathcal{Q}=\left(Q, \phi_{Q}\right)([4,16])$, is proved in [11] via the quantum affine Weyl-Schur duality functor $\mathcal{F}_{\mathcal{Q}}([10,12,17,21])$ from the category $\mathcal{C}_{Q H}$ of finite-dimensional graded modules over the symmetric quiver Hecke algebra to $\mathscr{C}_{\mathcal{Q}}$. More precisely, the category $\mathcal{C}_{Q H}$ provides a monoidal categorification of the quantum cluster algebra $A_{q}(\mathfrak{n})$, the quantum unipotent coordinate algebra of finite simply-laced type ([1]). Since $\mathcal{F}_{\mathcal{Q}}$ is an exact monoidal functor preserving simplicity, we can prove the conjecture for $\mathscr{C}_{\mathcal{Q}}$ in an indirect way. However, this method could not be applicable to other $\mathcal{C}$ directly.

Recently, in [13], the authors of the present paper (KKOP) developed $\mathbf{Z}$-valued invariants $\Lambda, \Lambda^{\infty}, \tilde{\Lambda}, \mathfrak{d}$ for pairs of modules in $\mathscr{C}_{\mathfrak{g}}$, which is extracted from distinguished $U_{q}^{\prime}(\mathfrak{g})$-module homomorphisms, called R-matrices. Furthermore, KKOP provided a criterion for a monoidal subcategory $\mathscr{C} \subset \mathscr{C}_{\mathfrak{g}}$ to become a monoidal categorification of a cluster algebra by using those invariants. This paper can be understood as a continuation of [13], since we will apply the above criterion to various subcategories $\mathscr{C}$ of $\mathscr{C}_{\mathfrak{g}}$, including $\mathscr{C}_{\mathfrak{g}}^{0}, \mathscr{C}_{\mathfrak{g}}^{-}$ 
and $\mathscr{C}_{N}$. We also give their initial monoidal seeds in a uniform manner.

Let $\mathfrak{g}_{0}$ be a finite-dimensional simple Lie algebra of $A D E$ type with a Cartan matrix $\mathrm{A}=$ $\left(a_{i j}\right)_{i, j \in I_{0}}$, W the Weyl group generated by simple reflections $s_{i}\left(i \in I_{0}\right), \mathfrak{g}$ the untwisted affine KacMoody algebra associated with $\mathfrak{g}_{0}$, and $U_{q}^{\prime}(\mathfrak{g})$ the quantum affine algebra associated with $\mathfrak{g}$. In [6], Hernandez-Leclerc defined the full subcategory $\mathscr{C}_{\mathfrak{g}}^{0}$ of $\mathscr{C}_{\mathfrak{g}}$. Since every simple modules in $\mathscr{C}_{\mathfrak{g}}$ is a tensor product of suitable parameter shifts of simple modules in $\mathscr{C}_{\mathfrak{g}}^{0}$, it is enough to consider subcategories of $\mathscr{C}_{\mathrm{g}}^{0}$.

By extending a reduced expression $s_{i_{1}} s_{i_{2}} \cdots s_{i_{\ell}}$ of the longest element $w_{0}$ of the Weyl group W, we obtain an infinite sequence

$$
\widehat{w}_{0}=\cdots s_{i_{-2}} s_{i_{-1}} s_{i_{0}} s_{i_{1}} s_{i_{2}} \cdots
$$

of simple reflections satisfying properties (a) and (b) in Section 2, and then we define fundamental modules $V[k]^{\hat{w}_{0}}(k \in \mathbf{Z})$. For each interval $[a, b]=$ $\{k \in \mathbf{Z} \mid a \leqslant k \leqslant b\}$ with $a \leqslant b \in \mathbf{Z} \sqcup\{ \pm \infty\}$, we define the subcategory $\mathscr{C}_{\mathfrak{g}}^{[a, b]}$ of $\mathscr{C}_{\mathfrak{g}}^{0}$ which is the smallest full monoidal subcategory containing $V[k]^{\widehat{w}_{0}}$ for all $k \in[a, b]$. Then $\mathscr{C}_{\mathfrak{g}}^{0}$ is nothing but $\mathscr{C}_{\mathfrak{g}}^{[-\infty,+\infty]}$ and the subcategory $\mathscr{\mathscr { C }}_{\mathfrak{g}}^{-}$introduced by Hernandez-Leclerc ([9]) can be identified with $\mathscr{C}_{\mathfrak{g}}^{[-\infty, 0]}$ (Remark 2.3).

We say that an interval $[a, b]$ is an $i$-box if $i_{a}=i_{b}$. For each $i$-box $[a, b]$, we define a simple module $M[a, b]$, which can be understood as a quantum affine analogue of the determinantial module (see Remark 3.2). In Theorem 3.1, we show that $M[a, b]$ is a Kirillov-Reshetikhin module and give a sufficient condition for the simplicity of the tensor product $M[a, b] \otimes M\left[a^{\prime}, b^{\prime}\right]$ for $i$-boxes $[a, b]$ and $\left[a^{\prime}, b^{\prime}\right]$. Then we define the notion of an admissible chain $\mathfrak{C}=\left\{\left[a_{k}, b_{k}\right] \subset[a, b] \mid 1 \leqslant k \leqslant b-\right.$ $\left.a+1, i_{a_{k}}=i_{b_{k}}\right\}$ of $i$-boxes for an interval $[a, b]$ satisfying certain properties (Definition 3.3). For each admissible chain $\mathfrak{C}$, the family of KirillovReshetikhin modules $M(\mathfrak{C})=\left\{M\left[a_{k}, b_{k}\right]\right\}_{1 \leqslant k \leqslant b-a+1}$ in $\mathscr{C}_{\mathfrak{g}}^{[a, b]}$ forms a commuting family of real simple modules (Theorem 3.4).

The next step is to show that $K\left(\mathscr{C}_{\mathfrak{g}}^{[a, b]}\right)$ has a cluster algebra structure, $\mathscr{C}^{[a, b]}$ provides a monoidal categorification of $K\left(\mathscr{C}_{\mathfrak{g}}^{[a, b]}\right)^{\mathfrak{g}}$, and any admissible chain $\mathfrak{C}$ gives a monoidal seed $M(\mathfrak{C})$ (Theorem 4.3). Based on the criterion in [13], we shall prove this by showing the assertion for a special chain $\mathfrak{C}$, and then by extending it to a general $\mathfrak{C}$. Namely, we proceed by proving

(i) the existence of a $\Lambda$-admissible monoidal seed $\mathscr{S}$ of $K\left(\mathscr{C}_{\mathfrak{g}}^{[a, b]}\right)$ whose set of initial cluster variable modules is $M(\mathfrak{C})$ for some admissible chain $\mathfrak{C}$,

(ii) the existence of sequences of mutations among the $M(\mathfrak{C})$ 's only employing $T$-systems,

which implies that any admissible chain $\mathfrak{C}$ gives a $\Lambda$-admissible monoidal seed for all $\mathscr{C}_{\mathfrak{g}}^{[a, b]}$. In particular, we prove that $\mathscr{C}$ is a monoidal categorification of the cluster algebra $K(\mathscr{C})$ for $\mathscr{C}=\mathscr{C}_{\mathfrak{g}}^{0}$ and $\mathscr{C}=\mathscr{C}_{\mathfrak{g}}^{-}$. Note that we need in step (i) above the existence of the cluster algebra structure on $K\left(\mathscr{C}_{\mathfrak{g}}^{-}\right)$ proved in [9].

This paper is an announcement whose details will appear elsewhere.

2. Subcategories. We take the algebraic closure $\mathbf{k}$ of $\mathbf{C}(q)$ inside $\bigcup_{m>0} \mathbf{C}\left(\left(q^{1 / m}\right)\right)$ as the base field for $U_{q}^{\prime}(\mathfrak{g})$. Recall that $\mathscr{C}_{\mathfrak{g}}$ is the category of finite-dimensional integrable modules over $U_{q}^{\prime}(\mathfrak{g})$. There is a family $\left\{V\left(\varpi_{i}\right)_{c} \mid i \in I_{0}, c \in \mathbf{k}^{\times}\right\}$in $\mathscr{C}_{\mathfrak{g}}$ of simple modules, called the fundamental modules.

For simple modules $M$ and $N$ in $\mathscr{C}_{\mathfrak{g}}$, we say that $M$ and $N$ strongly commute if $M \otimes N$ is simple, and $M$ is real if $M^{\otimes k}$ is simple for all $k \in \mathbf{Z}_{\geqslant 1}$.

Let us denote by $\Psi$ the quiver whose set of vertices is

$$
\widehat{I}_{0}:=\left\{(i, k) \in I_{0} \times \mathbf{Z} \mid k \equiv d(1, i) \bmod 2\right\} .
$$

and the arrows of $\Psi$ consist of two types:

$$
\begin{cases}(\mathrm{A}) \quad & (i, t) \rightarrow(j, s) \text { with } d(i, j)=1 \text { and } \\ & s-t=1 \\ (\mathrm{~B}) & (i, s+2) \rightarrow(i, s)\end{cases}
$$

Here $d(i, j)$ denotes the distance between the vertices $i$ and $j$ in the Dynkin diagram of $\mathfrak{g}_{0}$ and $1 \in I_{0}$ is an arbitrary chosen element.

We say that an infinite sequence

$$
\widehat{w}_{0}=\cdots s_{i_{-1}} s_{i_{0}} s_{i_{1}} \cdots
$$

of simple reflections in the Braid group $B\left(\mathfrak{g}_{0}\right)([15])$ of type $\mathfrak{g}_{0}$ is admissible if

(a) there exists a sequence $\left\{t_{k}\right\}_{k \in \mathbf{Z}}$ of integers such that
(1) $\left(i_{k}, t_{k}\right) \in \widehat{I}_{0}$,
(2) $t_{k^{+}}=t_{k}+2$, and
(3) $t_{k}>t_{k^{\prime}}$ if $k>k^{\prime}$ and $d\left(i_{k}, i_{k^{\prime}}\right)=1$. 
(b) $s_{i_{k}} \cdots s_{i_{k+\ell-1}}=w_{0}$ for all $k \in \mathbf{Z}$, where $\ell$ denotes the length of longest element $w_{0} \in \mathrm{W}$.

Here, for $k \in \mathbf{Z}$ and $j \in I_{0}$, we set

$$
\begin{aligned}
k^{+} & :=\min \left\{p \mid k<p, i_{k}=i_{p}\right\}, \\
k^{-} & :=\max \left\{p \mid p<k, i_{k}=i_{p}\right\}, \\
k(j)^{+} & :=\min \left\{p \mid k \leqslant p, i_{p}=j\right\}, \\
k(j)^{-} & :=\max \left\{p \mid p \leqslant k, i_{p}=j\right\} .
\end{aligned}
$$

\section{Remark 2.1.}

(i) We have $i_{k+\ell}=i_{k}^{*}$, where ${ }^{*}$ denotes the involution on $I_{0}$ induced by $w_{0}$.

(ii) $\widehat{w}_{0}$ completely determines $\left\{\left(i_{k}, t_{k}\right)\right\}_{k \in \mathbf{Z}}$ up to an even translation.

(iii) For every $k \in \mathbf{Z}$, the reduced expression $s_{i_{k}} \cdots s_{i_{k+\ell-1}}$ in (b) is adapted to some Dynkin quiver $Q$ of type $\mathfrak{g}_{0}$. Conversely, for any Dynkin quiver $Q$ of type $\mathfrak{g}_{0}$, there exists a sequence $\widehat{w}_{0}$ satisfying (a) and (b) such that $s_{i_{1}} \cdots s_{i_{\ell}}$ is adapted to $Q$.

For each $k \in \mathbf{Z}$, we define the fundamental module

$$
V[k]^{\widehat{w}_{0}}:=V\left(\varpi_{i_{k}}\right)_{(-q)^{t_{k}}} .
$$

Then we have

$$
V\left[k^{ \pm}\right]^{\widehat{w}_{0}} \simeq V[k]_{(-q)^{ \pm 2}}^{\widehat{w}_{0}}, \quad V[k+\ell]^{\widehat{w}_{0}}=\mathscr{D}\left(V[k]^{\widehat{w}_{0}}\right),
$$

where $\mathscr{D}$ denotes the right dual functor.

Definition 2.2. For each interval $[a, b]$, we denote by $\mathscr{C}_{\mathfrak{g}}^{[a, b]}$ the smallest full subcategory of $\mathscr{C}_{\mathfrak{g}}$ satisfying the following conditions:

(i) it is stable under taking subquotients, extensions, tensor products and

(ii) it contains $V[k]^{\hat{w}_{0}}$ for all $a \leqslant k \leqslant b$ and the trivial module $\mathbf{1}$.

Remark 2.3. Many of known subcategories $\mathcal{C}$ of $\mathscr{C}_{\mathfrak{g}}$ can be identified with $\mathscr{C}_{\mathfrak{g}}^{[a, b]}$ by taking suitable $\widehat{w}_{0}$ and $[a, b]$ :

(1) $\mathscr{C}_{\mathfrak{g}}^{[-\infty, \infty]}$ coincides with the subcategory $\mathscr{C}_{\mathfrak{g}}^{0}$.

(2) The subcategory $\mathscr{C}_{\mathcal{Q}}$ associated to a Q-data $\mathcal{Q}$ coincides with $\mathscr{C}_{\mathfrak{g}}^{[a, b]}$ for some interval $[a, b]$ with $|[a, b]|:=b-a+1=\ell$.

(3) By taking $s_{i_{1}} \cdots s_{i_{\ell}}$ in (b) as adapted to the Dynkin quiver $Q$ with $\{1,2\} \ni \phi_{Q}(k) \equiv d\left(1, i_{k}\right)$ $(\bmod 2)$ and $t_{k}=\phi\left(i_{k}\right)$ for $1 \leqslant k \leqslant\left|I_{0}\right|, \mathscr{C}_{N}$ can be identified with $\mathscr{C}_{\mathfrak{g}}^{[a, 0]}$ where $a=1-$ $\left(N \times\left|I_{0}\right|\right)$, and $\mathscr{C}_{\mathfrak{g}}^{-}$can be identified with $\mathscr{C}_{\mathfrak{g}}^{[-\infty, 0]}$. Those subcategories $\mathscr{C}_{N}, \mathscr{C}_{\mathfrak{g}}^{-}$of $\mathscr{C}_{\mathfrak{g}}^{0}$ are introduced in $[6,9]$.

3. Real simple commuting family associated to an admissible chain of $\boldsymbol{i}$-boxes. Let us fix an admissible sequence $\widehat{w}_{0}$ and $\left\{t_{k}\right\}_{k \in \mathbf{Z}}$. We write $V[k]$ for $V[k]^{w_{0}}$. We say that an interval $c=[a, b]$ is an $i$-box if $i_{a}=i_{b}$. For each $i$-box $[a, b]$, the module $M[a, b]$ in $\mathscr{C}_{\mathfrak{g}}^{0}$ is defined as follows:

$$
M[a, b]:=\operatorname{hd}\left(V[b] \otimes V\left[b^{-}\right] \otimes \cdots \otimes V\left[a^{+}\right] \otimes V[a]\right),
$$

where hd $(M)$ for $M \in \mathscr{C}_{\mathfrak{g}}$ denotes the head of $M$. In particular, $V[a]=M[a, a]$.

\section{Theorem 3.1.}

(i) $M[a, b]$ is a Kirillov-Reshetikhin module with $a$ dominant extremal weight $s \varpi_{i_{a}}$ where $s=$ $\left|\left\{k \mid a \leqslant k \leqslant b, i_{k}=i_{a}\right\}\right|$.

(ii) For $i$-boxes $[a, b]$ and $[c, d], M[a, b]$ and $M[c, d]$ commutes if either

$$
a^{-}<c \leqslant d<b^{+} \quad \text { or } \quad c^{-}<a \leqslant b<d^{+} .
$$

(iii) For any $i$-box $[a, b]$, there exists an exact sequence in terms of $M[a, b]$ 's as follows:

$$
\begin{gathered}
(3.1) 0 \rightarrow \underset{d\left(i_{a}, j\right)=1}{\otimes} M\left[a(j)^{+}, b(j)^{-}\right] \rightarrow M\left[a^{+}, b\right] \otimes \\
M\left[a, b^{-}\right] \rightarrow M[a, b] \otimes M\left[a^{+}, b^{-}\right] \rightarrow 0,
\end{gathered}
$$

We call it a T-system.

Remark 3.2. For any reduced expression $w_{0}=s_{j_{1}} \cdots s_{j_{\ell}}$ of $w_{0}$ (not necessarily adapted) and $\overline{[a}, b]$ with $j_{a}=j_{b}$ and $b-a+1 \leqslant \ell$, there exists a real simple module $\mathrm{D}[a, b]]^{w_{0}}$ in $\mathcal{C}_{Q H}$ of type $\mathfrak{g}_{0}$, called the determinantial module, and there exists an exact sequence (called the $T$-system)

$$
\begin{aligned}
0 \rightarrow \underset{d\left(i_{a}, j\right)=1}{\otimes} \mathrm{D}\left[a(j)^{+}, b(j)^{-}\right] & \rightarrow \mathrm{D}\left[a^{+}, b\right] \otimes \mathrm{D}\left[a, b^{-}\right] \\
& \rightarrow \mathrm{D}[a, b] \otimes \mathrm{D}\left[a^{+}, b^{-}\right] \rightarrow 0
\end{aligned}
$$

in $\mathcal{C}_{Q H}$, which is analogous to (3.1). More precisely, when $w_{0}$ is adapted to some Dynkin quiver $Q$ of type $\mathfrak{g}_{0}$, quantum affine Weyl-Schur duality functor $\mathcal{F}_{\mathcal{Q}}$ associated with some Q-data $\mathcal{Q}=\left(Q, \phi_{Q}\right)$ transforms the above exact sequence in $\mathcal{C}_{Q H}$ to the $T$-system (3.1). Thus $M[a, b]$ can be understood as a quantum affine analogue of the determinantial module. (See [7, Proposition 4.1] and [11] for more detail.)

For an interval $c:=[a, b]$, we introduce $i$-boxes

$$
\begin{aligned}
{[a, b\} } & :=\left[a, b\left(i_{a}\right)^{-}\right], \quad\{a, b]:=\left[a\left(i_{b}\right)^{+}, b\right], \\
\mathcal{L}(c) & :=[a-1, b\}, \quad \mathcal{R}(c):=\{a, b+1] .
\end{aligned}
$$

Definition 3.3. A chain $\mathfrak{C}$ of $i$-boxes

$$
\left(c_{k}=\left[a_{k}, b_{k}\right]\right)_{1 \leqslant k \leqslant l} \quad\left(l \in \mathbf{Z}_{\geqslant 1} \sqcup\{\infty\}\right)
$$

is called admissible if $\widetilde{c}_{k}=\left[\widetilde{a}_{k}, \widetilde{b}_{k}\right]:=\bigcup_{1 \leqslant j \leqslant k}\left[a_{j}, b_{j}\right]$ satisfies $\left|\widetilde{c}_{k}\right|=k$ and one of the following two statements. 
(1) $c_{k}=\mathcal{L}\left(\widetilde{c}_{k-1}\right)$,

(2) $c_{k}=\mathcal{R}\left(\widetilde{c}_{k-1}\right)$.

(Please do not confuse $l$ and $\ell$.) The sequence of intervals $\left\{\widetilde{c}_{k}\right\}_{1 \leqslant k \leqslant l}$ is called the envelope of the chain $\mathfrak{C}$ and $\widetilde{c}_{l}$ is called the range of $\mathfrak{C}$.

Thus, for an admissible chain $\mathfrak{C}$ of $i$-boxes, we can associate a pair $(a, \mathfrak{T})$ consisting of an integer $a$ and a sequence $\mathfrak{T}=\left(T_{1}, T_{2}, \ldots, T_{l-1}\right)$ such that

(i) $T_{i} \in\{\mathcal{L}, \mathcal{R}\}(1 \leqslant i \leqslant l-1)$,

(ii) $a=a_{1}=b_{1}$, and

(iii) $\left[\widetilde{a}_{k}, \widetilde{b}_{k}\right]= \begin{cases}{\left[\widetilde{a}_{k-1}-1, \widetilde{b}_{k-1}\right]} & \text { if } T_{k-1}=\mathcal{L}, \\ {\left[\widetilde{a}_{k-1}, \widetilde{b}_{k-1}+1\right]} & \text { if } T_{k-1}=\mathcal{R} .\end{cases}$

Hence we have $c_{k}=\left[a_{k}, b_{k}\right]=T_{k-1}\left[\widetilde{a}_{k-1}, \widetilde{b}_{k-1}\right](k \geqslant$ 2 ), and the interval $\widetilde{c}_{k}$ is obtained from $\widetilde{c}_{k-1}$ by adding an element from the left or from the right according that $T_{k-1}=\mathcal{L}$ or $T_{k-1}=\mathcal{R}$.

For an admissible chain $\mathfrak{C}=\left(c_{k}\right)_{1 \leqslant k \leqslant l}$ with the associated pair $(a, \mathfrak{T})$ and for $1 \leqslant s<l$, we say that an $i$-box $c_{s}$ is movable if $s=1$ or $T_{s-1} \neq T_{s}$ $(s \geqslant 2)$. For a movable $c_{s}$ in $\mathfrak{C}$, we define a new admissible chain $B_{s}(\mathfrak{C})$ whose associated pair $\left(a^{\prime}, \mathfrak{T}^{\prime}\right)$ is given

(i) $\begin{cases}a^{\prime}=a \pm 1 & \text { if } s=1 \text { and } T_{1}=\mathcal{R}(\text { resp. } \mathcal{L}) \text {, } \\ a^{\prime}=a & \text { if } s>1,\end{cases}$

(ii) $T_{k}^{\prime}=T_{k}$ for $k \notin\{s-1, s\}$, and

(iii) $T_{k}^{\prime} \neq T_{k}$ for $k \in\{s-1, s\}$.

That is, $B_{s}(\mathfrak{C})$ is the admissible chain obtained from $\mathfrak{C}$ by moving $\widetilde{c}_{s}$ by 1 to the right or to the left inside $\widetilde{c}_{s+1}$.

Theorem 3.4. Let $\mathfrak{C}=\left(c_{k}\right)_{1 \leqslant k \leqslant l}$ be an admissible chain and set

$$
M(\mathfrak{C}):=\left\{M\left[a_{k}, b_{k}\right] \mid 1 \leqslant k \leqslant l\right\} .
$$

Then we have the followings

(a) $M(\mathfrak{C})$ is a real simple commuting family.

(b) If $M[c, d]$ commutes with all $M\left[a_{k}, b_{k}\right]$ and $[c, d] \subset\left[\widetilde{a}_{l}, \widetilde{b}_{l}\right]$, then $[c, d] \in \mathfrak{C}$.

(c) For another admissible chain $\mathfrak{C}^{\prime}=\left(c_{k}^{\prime}\right)_{1 \leqslant k \leqslant l}$ with the same range, there exists a finite sequence $\left(t_{1}, t_{2}, \ldots, t_{r}\right) \in\{1,2, \ldots, l\}^{r}$ such that

$$
B_{t_{r}}\left(\cdots\left(B_{t_{2}}\left(B_{t_{1}}(\mathfrak{C})\right) \cdots\right)=\mathfrak{C}^{\prime} .\right.
$$

4. Monoidal categorification. Let $K=$ $K_{\text {ex }} \sqcup K_{\text {fr }}$ be a countable index set. Let $\mathscr{C}$ be a full subcategory of $\mathscr{C}_{\mathfrak{g}}^{0}$ stable under taking subquotients, extensions and tensor products.
A monoidal seed in $\mathscr{C}$ is a pair $\mathscr{S}=$ $\left(\left\{M_{i}\right\}_{i \in K}, \widetilde{B}\right)$ consisting of a commuting family $\left\{M_{i}\right\}_{i \in K}$ of real simple objects in $\mathscr{C}$ and a $\mathbf{Z}$-valued $K \times K_{\text {ex }}$-matrix $\widetilde{B}=\left(b_{i j}\right)_{(i, j) \in K \times K_{\text {ex }}}$ such that (i) for each $j \in K_{\mathrm{ex}}$, there exist finitely many $i \in K$ such that $b_{i j} \neq 0$, (ii) the principal part $B:=\left(b_{i j}\right)_{i, j \in K_{\mathrm{ex}}}$ is skew-symmetric. For $i \in K$, we call $M_{i}$ the $i$-th cluster variable module of $\mathscr{S}$.

For a monoidal seed $\mathscr{S}=\left(\left\{M_{i}\right\}_{i \in K}, \widetilde{B}\right)$, let $\Lambda^{\mathscr{S}}=\left(\Lambda_{i j}^{\mathscr{S}}\right)_{i, j \in K}$ be the skew-symmetric matrix given by $\Lambda_{i j}^{\mathscr{S}}=\Lambda\left(M_{i}, M_{j}\right)$ (see [14]).

A monoidal seed $\mathscr{S}=\left(\left\{M_{i}\right\}_{i \in K}, \widetilde{B}\right)$ is called $\Lambda$-admissible if

(i) $\left(\Lambda^{\mathscr{S}} \widetilde{B}\right)_{j k}=-2 \delta_{j k}$ for $(j, k) \in K \times K_{\mathrm{ex}}$, and

(ii) for each $k \in K_{\mathrm{ex}}$, there exist a simple object $M_{k}^{\prime}$ of $\mathcal{C}$ commuting with $M_{i}$ for any $i \neq k$ and an exact sequence in $\mathscr{C}$

$$
\begin{aligned}
0 \rightarrow \bigotimes_{b_{i k}>0} M_{i}^{\otimes b_{i k}} & \rightarrow M_{k} \otimes M_{k}^{\prime} \\
& \rightarrow \bigotimes_{b_{i k}<0} M_{i}^{\otimes\left(-b_{i k}\right)} \rightarrow 0 .
\end{aligned}
$$

Under the following two assumptions

(a) there exists a $\Lambda$-admissible monoidal seed $\mathscr{S}=\left(\left\{M_{i}\right\}_{i \in K}, \widetilde{B}\right)$ in $\mathscr{C}$,

(b) $K(\mathscr{C})$ is isomorphic to the cluster algebra $\mathscr{A}([\mathscr{S}])$,

KKOP $([13$, Theorem 6.10]) proved that $\mathscr{C}$ provides a monoidal categorification of $\mathscr{A}([\mathscr{S}])$. Here $[\mathscr{S}]:=\left(\left[\left\{M_{i}\right]\right\}_{i \in K}, \widetilde{B}\right)$ is a seed in $K(\mathscr{C})$, and $\mathscr{A}([\mathscr{S}])$ denotes the cluster algebra with the initial seed $[\mathscr{S}]$.

Set $\widehat{I}_{0}^{-}:=\widehat{I}_{0} \cap\left(I_{0} \times \mathbf{Z}_{\leqslant 0}\right)$ and let $\Psi^{-}$be the full subquiver of $\Psi$ whose set of vertices is $\widehat{I}_{0}^{-}$. In [9], Hernandez-Leclerc proved that $\mathscr{A}^{-}:=K\left(\mathscr{C}_{\mathfrak{g}}^{-}\right)$has a cluster algebra structure whose initial cluster variable modules $\left\{\mathcal{M}_{(i, t)}\right\}_{(i, t) \in \widehat{I}_{0}^{-}}$consist of certain KRmodules. For a suitable choice of $\widehat{w}_{0}$ (Remark 2.3), we have $\mathscr{C}_{\mathfrak{g}}^{-}=\mathscr{C}_{\mathfrak{g}}^{[-\infty, 0]}$ and $\left\{\mathcal{M}_{(i, t)}\right\}_{(i, t) \in \widehat{I}_{0}^{-}}$can be described as $M\left(\mathfrak{C}^{-}\right)$for the following admissible chain $\mathfrak{C}^{-}$of $i$-boxes:

$$
\mathfrak{C}^{-}=(0, \mathfrak{T}=(\mathcal{L}, \mathcal{L}, \mathcal{L}, \ldots)) .
$$

More precisely, for $(i, t)=\left(i_{a}, t_{a}\right)(a \leqslant 0)$, we have

$$
\mathcal{M}_{(i, t)}=M[a, 0\} .
$$

The following theorem gives an affirmative answer for the conjecture on $\mathscr{C}_{\mathfrak{g}}^{-}$:

Theorem 4.1. The monoidal seed 


$$
\mathscr{S}^{-}:=\left(M\left(\mathfrak{C}^{-}\right), \widetilde{B}^{-}\right) \text {is } \Lambda \text {-admissible, }
$$

where $\widetilde{B}^{-}$is the matrix associated to $\Psi^{-}$. Hence $\mathscr{C}_{\mathfrak{g}}^{-}$ provides a monoidal categorification of $\mathscr{A}^{-}$.

Now we shall generalize the above theorem to an arbitrary $\mathscr{C}_{\mathfrak{g}}^{[a, b]}$.

Proposition 4.2. Let $\mathfrak{C}=\left(c_{k}\right)_{1 \leqslant k \leqslant l}$ be an admissible chain of $i$-boxes with the range $[a, b]$ and the envelope $\left\{\widetilde{c}_{k}\right\}_{1 \leqslant k \leqslant l}$. Assume that $\mathscr{C}:=\mathscr{C}_{\mathfrak{g}}^{[a, b]}$ provides a monoidal categorification of $K(\mathscr{C})$ with a $\Lambda$-admissible monoidal seed $(M(\mathfrak{C}), \widetilde{B})$. Let $c_{s}$ be a movable $i$-box of $\mathfrak{C}$ and set $\mathfrak{C}^{\prime}=B_{s}(\mathfrak{C})$. If $\widetilde{c}_{s+1} \neq c_{s+1}$, then $M\left(\mathfrak{C}^{\prime}\right)$ is equal to $M(\mathfrak{C})$ up to a permutation. If $\widetilde{c}_{s+1}=c_{s+1}$, then $M\left(\mathfrak{C}^{\prime}\right)$ is the monoidal mutation of $M(\mathfrak{C})$ at $s$. Moreover the corresponding exact sequence (4.1) is given by the T-system (3.1).

The above proposition and Theorem 3.4 show that all $M(\mathfrak{C})$ with the same range are mutation equivalent.

Now we state our main theorem:

Theorem 4.3. For any admissible chain $\mathfrak{C}=$ $\left(c_{k}\right)_{1 \leqslant k \leqslant l}$ for $l \in \mathbf{Z}_{\geqslant 1} \sqcup\{\infty\}$ with the range

$$
\widetilde{c}_{l}=[a, b] \quad \text { for } a \leqslant b \in \mathbf{Z} \sqcup\{ \pm \infty\},
$$

there exists a $\Lambda$-admissible monoidal seed $\mathscr{S}$ of $\mathscr{C}_{\mathfrak{g}}^{[a, b]}$ such that

(i) its set of cluster variable modules is $M(\mathfrak{C})$,

(ii) its set of frozen variable modules is $\left\{M\left[a(i)^{+}, b(i)^{-}\right] \mid i \in I_{0},-\infty<a(i)^{+} \leqslant b(i)^{-}<\right.$ $+\infty\}$, and

(iii) $K\left(\mathscr{C}_{\mathfrak{g}}^{[a, b]}\right)$ has a cluster algebra structure with the initial seed $[\mathscr{S}]$, and $\mathscr{C}_{\mathfrak{g}}^{[a, b]}$ provides a monoidal categorification of $\mathscr{A}([\mathscr{S}]) \simeq$ $K\left(\mathscr{C}_{\mathfrak{g}}^{[a, b]}\right)$.

By Remark 2.3, we have the following

Corollary 4.4. The Grothendieck ring $K\left(\mathscr{C}_{\mathfrak{g}}^{0}\right)$ has a cluster algebra structure, and $\mathscr{C}_{\mathfrak{g}}^{0}$ provides a monoidal categorification of $K\left(\mathscr{C}_{\mathfrak{g}}^{0}\right)$.

Remark 4.5. We can generalize the above results to an arbitrary quantum affine algebra $U_{q}^{\prime}(\mathfrak{g})$ by applying a similar framework with the results in $[12,17,21,22]$.

Acknowledgements. The research of Kashiwara was supported by Grant-in-Aid for Scientific Research (B) 15H03608, Japan Society for the Promotion of Science, the research of Kim and $\mathrm{Oh}$ were supported by the National Research Foundation of Korea (NRF) Grant funded by the Korea government (MSIP) (NRF2017R1C1B2007824, NRF-2019R1A2C4069647).

\section{References}

[ 1 ] A. Berenstein and A. Zelevinsky, Quantum cluster algebras, Adv. Math. 195 (2005), no. 2, 405455 .

[ 2 ] S. Fomin and A. Zelevinsky, Cluster algebras. I. Foundations, J. Amer. Math. Soc. 15 (2002), no. 2, 497-529.

[ 3 ] E. Frenkel and N. Reshetikhin, The $q$-characters of representations of quantum affine algebras and deformations of $W$-algebras, in Recent developments in quantum affine algebras and related topics (Raleigh, NC, 1998), 163-205, Contemp. Math., 248, Amer. Math. Soc., Providence, RI, 1999.

[ 4 ] R. Fujita and S.-j. Oh, Combinatorics of twisted Auslander-Reiten quivers and Representations of quantum affine algebras, in preparation.

[ 5 ] D. Hernandez, The Kirillov-Reshetikhin conjecture and solutions of $T$-systems, J. Reine Angew. Math. 596 (2006), 63-87.

[6 ] D. Hernandez and B. Leclerc, Cluster algebras and quantum affine algebras, Duke Math. J. 154 (2010), no. 2, 265-341.

[ 7 ] D. Hernandez and B. Leclerc, Quantum Grothendieck rings and derived Hall algebras, J. Reine Angew. Math. 701 (2015), 77-126.

[ 8 ] D. Hernandez and B. Leclerc, Monoidal categorifications of cluster algebras of type $A$ and $D$, in Symmetries, integrable systems and representations, 175-193, Springer Proc. Math. Stat., 40, Springer, Heidelberg, 2013.

[ 9 ] D. Hernandez and B. Leclerc, A cluster algebra approach to $q$-characters of Kirillov-Reshetikhin modules, J. Eur. Math. Soc. (JEMS) 18 (2016), no. 5, 1113-1159.

[ 10 ] S.-J. Kang, M. Kashiwara and M. Kim, Symmetric quiver Hecke algebras and $R$-matrices of quantum affine algebras, II, Duke Math. J. 164 (2015), no. 8, 1549-1602.

[11 S.-J. Kang, M. Kashiwara, M. Kim and S.-j. Oh, Monoidal categorification of cluster algebras, J. Amer. Math. Soc. 31 (2018), no. 2, 349-426.

[12] M. Kashiwara, M. Kim, S.-j. Oh and E. Park, Symmetric quiver Hecke algebras and $R$-matrices of quantum affine algebras IV, Selecta Math. (N.S.) 22 (2016), no. 4, 1987-2015.

[13 ] M. Kashiwara, M. Kim, S.-j. Oh and E. Park, Monoidal categorification and quantum affine algebras, Compos. Math. 156 (2020), no. 5, 1039-1077.

[ 14 ] M. Kashiwara, M. Kim, S.-j. Oh and E. Park, Block decomposition for quantum affine algebras by the associated simply-laced root system, arXiv:2003.03265v1.

[15 ] M. Kashiwara, M. Kim, S.-j. Oh and E. Park, Braid group action on the module category of quantum affine algebras, Proc. Japan Acad. Ser. A Math. Sci. 97 (2021), no. 3, 13-18.

[16 ] M. Kashiwara, M. Kim, S.-j. Oh and E. Park, PBW theoretic approach to the module category of quantum affine algebras, Proc. Japan 
Acad. Ser. A Math. Sci. 97 (2021), no. 6, 3337.

[ 17 ] M. Kashiwara and S.-j. Oh, Categorical relations between Langlands dual quantum affine algebras: doubly laced types, J. Algebraic Combin. 49 (2019), no. 4, 401-435.

[18 ] A. Kuniba, T. Nakanishi and J. Suzuki, Functional relations in solvable lattice models. I. Functional relations and representation theory, Internat. J. Modern Phys. A 9 (1994), no. 30, $5215-5266$.

[ 19 ] H. Nakajima, Quiver varieties and finite-dimensional representations of quantum affine algebras, J. Amer. Math. Soc. 14 (2001), no. 1, 145238 .
[ 20 ] H. Nakajima, Quiver varieties and cluster algebras, Kyoto J. Math. 51 (2011), no. 1, 71-126.

[21 ] S.-j. Oh and T. Scrimshaw, Categorical relations between Langlands dual quantum affine algebras: exceptional cases, Comm. Math. Phys. 368 (2019), no. 1, 295-367.

[ 22 ] S.-j. Oh and U. R. Suh, Twisted and folded Auslander-Reiten quivers and applications to the representation theory of quantum affine algebras, J. Algebra 535 (2019), 53-132.

[23 ] F. Qin, Triangular bases in quantum cluster algebras and monoidal categorification conjectures, Duke Math. J. 166 (2017), no. 12, 23372442 . 